\title{
MATHEMATICAL MODEL FOR ICE WALL INTERACTIONS WITHIN A LEVEL SET METHOD
}

\section{F. Morency ${ }^{*}$}

École de technologie supérieure, 1100 rue Notre-Dame Ouest, Montréal, QC, Canada, H3C 1K3, email:francois.morency@etsmtl.ca

\section{H. Beaugendre}

Université de Bordeaux, IMB, UMR 5251, F-33400 Talence, France,

CNRS, IMB, UMR 5251, F-33400 Talence, France

Bordeaux INP, IMB, UMR 5251, F-33400 Talence, France

INRIA, F-33400 Talence, France

Email: heloise.beaugendre@math.u-bordeaux.fr

* corresponding author

\begin{abstract}
De-icing systems work periodically to remove ice formed on aircraft protected surfaces. They achieve this result by reducing adhesive shear strength between ice and surfaces. In the present work, an immersed boundary method will predict ice-shedding trajectories and collisions. At the starting-point of shedding trajectory calculations, when the ice-shed particle is still close to the wing surface, there is a high probability of collision with the surface. A linear spring collision model is presented assuming the interacting bodies are connected tangentially and normally by linear springs. The verification of the model is made by comparison with literature results and study of ice trajectories around a cylinder and an aerofoil.
\end{abstract}

Keywords : ice wall interactions, level set methods, mathematical models, immersed boundary methods, ice shedding, trajectories, collisions, linear spring models, cylinder, aerofoil

Biographical notes: François Morency is an associate professor at the department of mechanical engineeering at École de Technologie Supérieure(ÉTS). He teaches fluid dynamics, thermodynamics, and aircraft design courses. His areas of interest in research include aircraft ice protection system design; human factors in aircraft ground de-icing; and air quality in workplace environment. He has completed is $\mathrm{PhD}$ in mechanical engineering at École Polytechnique de Montréal in 1999. 
Héloïse Beaugendre has a Phd from McGill University devoted to the development of a modeling tool for ice accretion on complex geometries. Assistant professor at the mathematical institute of Bordeaux and member of CARDAMOM Inria team to propose efficient numerical tools to predict ice shedding trajectories. Member of the European project STORM FP7 dedicated to "Efficient ice protection Systems and simulation Techniques Of ice Release on propulsive systeMs" - STORM

\section{INTRODUCTION}

Aerodynamic and environment are tightly bound together since the early days of aviation. Inflight, except for the pilot competencies, aircraft performances depend mostly on aerodynamic, structural, control, and propulsion characteristics. The environment can affect each of these characteristics, but, arguably, aerodynamic is at the heart of aircraft design. Atmospheric turbulence and weather conditions can adversely affect aircraft aerodynamic and lead to an accident, causing injury, or death to passengers or aircraft crew members.

According to a report of the United-States National Transportation Safety Board (2011), between 2007 and 2009, atmospheric turbulence encounters during the en route phase of flight was the main cause of accidents on commercial flight. Flying in adverse weather conditions is always dangerous, especially weather conditions leading to ice accretion on airframe that pose a significant hazard for aircraft operation in the Northern Hemisphere. According to a survey of the National Transport Safety Board online database and NASA Aviation Safety Reporting System from 1978 to 2005 (Green, 2006), 944 accidents and incidents can be imputed to inflight icing. It gives an average of thirty-five accidents/year. A following study of aircraft icing accidents and incidents using the same databases, but for the period 2006 to 2010, found 258 accidents or incidents caused by inflight icing (Appiah-Kubi, 2011), an average of sixty-four accidents/year. The increase in yearly accident rate is must probably caused by the increase in total flight hours since 1978. Ice accretions cause most of the time aerodynamic performance degradations that lead to aircrafts stall or loss of control. 
Several measures can help to reduce aircraft icing hazard: meteorological prediction to help en route aircraft to avoid icing events, increase pilot awareness to icing danger through formations, install efficient ice protection system (IPS) on aircraft critical part. An aircraft can only fly in icing condition if IPSs are installed on critical part such as leading edge of wing and stabilizers. Anti-icing type IPS works continuously during an icing event, preventing all ice accretion in the critical area. The surface is usually heated such that i) either all the water caught on a critical part evaporates (evaporative mode) or ii) the water partially evaporates and runs back, freezing on a less critical area (wet mode). De-icing type IPS works periodically, waiting for a small amount of ice to accrete before removing it.

De-icing systems require less energy than anti-icing systems. For example, in a NASA study (Miller et al., 1997), an electrical IPS was installed on a wing leading edge and used in anti-icing and de-icing mode for the same ice accretion conditions. The goal of this study was to build an experimental database to validate a numerical method, but energy consumption can be computed from their data for two icing conditions. The tests were run in a wind tunnel. For a total air temperature of $-6.7^{\circ} \mathrm{C}$, a velocity of $44.7 \mathrm{~m} / \mathrm{s}$, a liquid water content of $0.78 \mathrm{~g} / \mathrm{m}^{3}$, and a median volumetric droplet diameter of $20 \mu \mathrm{m}$, the energy consumption by meter wing span is $10.3 \mathrm{~kW}$ for the anti-icing system running in evaporative mode, $1.89 \mathrm{~kW}$ for the anti-icing system running in wet mode (Al-Khalil et al., 1997). With the same ambient conditions, two de-icing system configurations are tested and the energy consumption is $1.05 \mathrm{~kW}$ or $0.940 \mathrm{~kW}$ (Wright, Al-Khalil and Miller, 1997). For a colder total air temperature $-17.8^{\circ} \mathrm{C}$, the evaporative mode uses $11.4 \mathrm{~kW}$, the running wet mode uses 3.53 $\mathrm{kW}$ and the de-icing system configurations use $1.64 \mathrm{~kW}$ or $2.15 \mathrm{~kW}$. Thus, waiting for some ice to accrete reduces the IPS energy consumption by a factor between ten and five. This energy reduction is very attractive for engineers that seek to produce green aircraft.

De-icing systems remove ice formed on aircraft protected surfaces according to a periodic cycle by reducing the adhesive force between ice and surface. Once the adhesive force is reduced, aerodynamic forces blow the ice at a distance from the surface. The use of aircraft de-icing system raises the need to predict ice-shedding trajectories to allow assessing the risk of impact/ingestion on/in aircraft components located downstream. Aircraft manufacturers rely mainly on flight tests to evaluate the potential negative effects of ice shedding because of the lack of applicable numerical tools. It is difficult for classical CFD tools to predict trajectories because of the shapes and sizes randomness of the ice-shed particles. 
In the present work, the motion of the solids (ice blocks) is computed using the basic physical laws (CFD) meaning that no database has to be used to predict drag and the lift imposed on ice pieces. The use of level set, and penalisation methods enable a tight coupling between airflow and ice pieces. The tight coupling involves considering the ice piece effects on the airflow, contrary to classical method, and the corresponding airflow effects on the ice piece, the usual drag and lift forces. This numerical method has been used successfully to predict bluff body motions in a previous study (Beaugendre et al., 2011). However, this study showed that a collision model was needed to consider the ice block interactions with the wall at the starting-point of the shedding phenomena. The goal of the present paper is to develop a collision force model that will enable the ice pieces to rebound on the aircraft skin into the airflow, considering forces in normal and tangential direction.

Some models used with CFD calculation in the literature are first reviewed. Next, the three most important collision parameters are presented and typical values for ice bouncing in an aircraft de-icing situation are calculated. Then, the normal and tangential linear spring model proposed to model bouncing is presented. An applicable numerical method is afterward proposed to use the linear spring model within the framework of level set functions. The model implementation is verified with the test case of a sphere moving at constant speed into an empty space, impacting a wall at various angles. Then, an ice shape impinging a cylinder and an aerofoil wall displaced by aerodynamic forces will be studied.

\section{LITTERATURE REVIEW}

In numerical flow simulations, when two bodies overlap, a collision is considered to occur. Most of the viscous flow simulation past moving rigid bodies proposes simple collision models. Glowinski et al. (2001) propose a strategy to consider collision based on a short range repulsive force. The repulsive force acts in the normal direction, and it takes a finite value depending on the distance between particle centres. The force starts acting when the space between the two particle surfaces is small but positive, to ensure that no overlap occurs. Singh, Hesla and Joseph (2003), and Wan and Turek (2006) present numerical schemes that allow particles to overlap slightly. A repulsive force is introduced to ensure overlapping does not exceed one element. The force acts also in the normal direction, and is proportional to a stiffness parameter times the distance between particle centres. Patankar and Sharma (2005) 
use the same collision force definition, but apply it in an immersed boundary method based on a fractional time stepping strategy. Sub-steps in the position update are used during the collision process to improve accuracy. The repulsive force methods do not guarantee that the particles will not overlap, and the rebound velocity depends strongly on a stiffness parameter, not easily linked to material properties.

Another model is proposed by Ardekani and Rangel (2007) to avoid these two weakness' of repulsive force methods. The model is based on conservation of linear momentum along the line between the centres of the two colliding spherical particles. The normal restitution coefficient along the line between the centres depends on the Stokes number. The linear momentum in the tangential direction and the angular velocity are conserved for each particle implied in the collision. However, the extension of the model to solids of general shapes needs further investigation.

The use of level set method opens the possibility to define a repulsive force based on distance between solid boundaries instead of solid centres. Coquerelle and Cottet (2008) use a repulsive force model based on a singular function for the calculation of the repulsive force. The repulsive force becomes infinite as the distance between the surfaces tends to 0 , to avoid overlap between particles. However, the repulsive force acts only in the normal direction, neglecting tangential forces occurring when tangential velocities at solid boundaries are not equal prior to collision.

Tangential forces are particularly important when collision angles are far from normal direction, a situation that occurs frequently for collisions at the starting-point of the shedding phenomena. The model proposed in the present paper came from the discrete element method field in which various models have been implemented to cater with prediction of granular flow, with spherical bodies of known radius (Thornton, Cummins and Cleary, 2011). A commonly used model is the linear spring-dashpot model (Cleary and Sawley, 2002). A further simplification of the model is to neglect the dashpot, thus assuming collision is elastic with no dissipation.

\section{COLLISION PARAMETERS}

In multiphase flows, when solid particles are free to move, particle-wall interactions play a major role in the dynamic of the flow. For example, in aircraft de-icing CFD simulations, shed ice particles must be kept outside the wing by a specific model for these interactions. 
The bouncing of a solid sphere on a wall in air or in various fluids as been well studied. The studies have shown that the restitution coefficient, the ratio of the velocity after the rebound to the approach velocity, depends on the particle Stokes number St:

$$
S t=\frac{\text { particle inertia }}{\text { viscous effects }}
$$

The restitution coefficient of two colliding objects is a number between 0.0 and 1.0 representing the ratio of velocities after and before a collision. Below a critical value for the Stokes number, around 10, a no rebound situation occurs, such that the restitution coefficient $e=0$ (Legendre et al., 2006). At the other extremity, for $S t>10^{4}$, the viscous effects are supposed to be negligible and the restitution coefficient reach an asymptotic value close to one for elastic material. Between these two values, the restitution coefficient increases monotonically. It is important to notice however that for viscous collision, the velocity starts slowing down before the particle touches the wall, leading to a modified definition of the restitution coefficient:

$$
e=-\frac{V_{r e s}}{V_{\infty}}
$$

where $V_{\text {res }}$ is the velocity after the rebound and $V_{\infty}$ is the velocity before its decrease resulting from the wall interaction.

For spherical particles, the Stokes number is defined as

$$
\mathrm{St}=\frac{\left(\rho_{p}+0.5 \rho\right) V_{\infty} D}{9 \mu}
$$

where $\rho_{p}$ is the particle density, $\rho$ is the fluid density, and $\mu$ the fluid viscosity. The restitution coefficient for collision in air is given by 


$$
e=0.91 \exp \left[-\frac{35}{\mathrm{St}}\right]
$$

according to Legendre et al. (2006).

Another important parameter for bouncing model is the contact time. The Hertzian theory gives the following relation for solid sphere in gas bouncing on a wall

$$
t_{\text {Hertz }}=2.54 \rho_{p}^{2 / 5}\left[\frac{1-v_{p}^{2}}{E_{p}}+\frac{1-v_{w}^{2}}{E_{w}}\right]^{2 / 5} D V_{i m p}^{-1 / 5}
$$

where $E$ and $v$ are the modulus of elasticity and the Poisson's ratio and the sub-indices $w$ and $p$ refer to the wall and the particle.

\subsection{Ice particle in air}

The three parameters defined by Equations 3, 4, and 5 can be estimated if ice and airflow properties are known around the aircraft wing.

There are several types of microstructure possible for ice, depending on the physical scales of the ice studied, the temperature, the pressure, the way it forms or if its ground or atmospheric ice (Coles, 2001). It is a challenging task for the engineer to estimate the mechanical properties of ice. For the present work, three properties are of concern, the density, the elastic modulus and the Poisson's ratio. For ice plates at $-10^{\circ} \mathrm{C}$, the Young's modulus of ice is in the range 9.7-11.2 GPa and the Poisson's ratio is between 0.29 and 0.32 (Petrovic, 2003). According to experimental measurements of Gammond et al. (1983) on ground ice samples, at $-16^{\circ} \mathrm{C}$, the ice density is $919.5 \mathrm{~kg} / \mathrm{m}^{3}$, the Young's modulus is 9.332 $\mathrm{GPa}$ and the Poisson's ratio is 0.325 .

Consider a large ice accretion removed by aerodynamic forces, a situation that can occur if no ice protection system is used and the aircraft simply flies out of in-flight icing conditions. For an ice particle of around $5 \mathrm{~cm}$ diameter, impinging on an aluminum wall (density $2796 \mathrm{~kg} / \mathrm{m}^{3}$, Young's modulus $71.7 \mathrm{GPa}$, Poisson's ratio 0.33), moving at a velocity of $30 \mathrm{~m} / \mathrm{s}$ in air (density $1.23 \mathrm{~kg} / \mathrm{m}^{3}$, viscosity $1.9 \times 10^{-5} \mathrm{~kg} / \mathrm{m} \mathrm{s}$ ), St is around $8 \times 10^{6}$. For this high value of St, the value of the restitution coefficient is around 0.91 . The contact time is in the order of magnitude of $10^{-4} \mathrm{~s}$. 
Using a Vortex In Cell (VIC) scheme for the CFD solution, see Beaugendre et al. (2011), the calculation time step depends mostly on the cell size and viscosity. If the time step used is equal or larger than the contact time, this may lead to non-physical results, where the restitution coefficient is larger than one. In such situation, the contact time is smaller than the viscous relaxation time and the collision can be modeled as a discontinuity (Legendre et al., 2006), without considering the effect on airflow. The other possibility is to reduce the calculation time step to ensure that the collision phenomenon is discretized with at least ten time steps (Patankar and Sharma, 2005).

\section{COLLISION FORCE MODEL}

Consider the collision of a spherical body of diameter D with a wall, as shown on figure 1 . The sphere is moving with a velocity $V_{i}$ prior to the contact, without rotation. The sphere collides with the wall at point 0 . Under the effects of collision forces, the sphere rebounds with a velocity $V_{r}$ and an angular velocity $\omega_{r}$. The angle between the rebound velocity vector with the vertical axe, $\theta_{r}$, may differ from the angle between the impact velocity vector and the vertical axis, $\theta_{i}$. The model allows body overlapping during the contact time. The sphere overlaps with the wall on a distance $\alpha$ in the normal direction and the relative tangential displacement from the collision point is $\delta$.

For the linear spring model, the two interacting bodies are connected normally and tangentially by linear springs. The normal and tangential forces, $F_{n}$ and $F_{t}$ during collision are calculated with the following equations:

$$
\begin{gathered}
F_{n}=k_{n} \alpha \\
F_{t}^{\text {new }}=\left\{\begin{array}{cc}
F_{t}^{\text {old }}+k_{t} \Delta \delta & \text { if } F_{t}^{\text {new }}<\mu F_{n} \\
\mu F_{n} & \text { if } F_{t}^{\text {new }} \geq \mu F_{n}
\end{array}\right.
\end{gathered}
$$

where $k_{n}$ and $k_{t}$ are the normal and tangential spring stiffness, and $\mu$ is the interface friction coefficient. 
The normal spring stiffness depends on the mass $m$ of the body, the normal impact velocity $V_{n i}$, the sphere radius $R$, and a ratio $E^{*}$, function of Young's modulus $E$ and Poisson's ratio $v$. For a sphere, Thornton, Cummins and Cleary (2011) proposed an equation based on the theory of Hertz :

$$
k_{n}=1.2024\left(m^{1 / 2} E^{* 2} R V_{n i}\right)^{2 / 5}
$$

where

$$
E^{*}=\frac{E}{2\left(1-v^{2}\right)}
$$

The ratio of tangential spring stiffness over the normal spring stiffness relates the value for $k_{t}$ to the Poisson's coefficient:

$$
\kappa=\frac{k_{t}}{k_{n}}=\frac{2(1-v)}{2-v}
$$

The normal spring stiffness controls the overlap accepted between the two bodies and the duration of the collision. A stiffer spring means a shorter collision time. A softer spring means a longer collision time and more overlap.

\section{NUMERICAL METHOD}

The collision force model is implemented within a code presented earlier in Beaugendre et al. (2011). The code solves the Navier-Stokes equations around rigid bodies in motion using an immersed boundary method. The solid domains are defined using level sets function, and a penalisation method enforces rigid velocity inside the solid. A vortex in cell scheme (VIC) solves the flow field on a Cartesian grid (Coquerelle and Cottet, 2008).

With level set methods, each body is defined with a function $\phi$ that contains the sign normal distance from the body surface, the distance being negative inside the body. At each body-fluid interface, the level set function takes a value of zero. The figure 2 illustrates the situation for a circular ice piece, $\phi_{i c e}$, in contact with an aerofoil wall, $\phi_{\text {wing }}$. All the 
information required by the collision model is already available within the code, but a collision force subroutine must be added and integrated within the time splitting algorithm.

\subsection{Collision force and level set function}

Consider the two bodies defined by the level set functions $\phi_{i c e}$ and $\phi_{\text {wing }}$ in a domain discretized by a Cartesian grid illustrated on figure 2. Each node of the grid stores the values of the two level set functions. The two bodies are in contact if $\phi_{i c e}<0$ and $\phi_{\text {wing }}<0$ for some nodes of the grid. On figure 2 , the nodes $(i-2, j),(i-1, j),(i, j),(i+1, j)$, and $(i+2, j)$ are in this situation.

If there is a contact, a repulsive force must be computed. Figure 3 defines the variables needed for the normal and tangential force calculations. The normal distance deformation $\alpha$ is given by the level set function, $\phi_{i c e}$, that is the negative distance between a point and the body surface. Here, there is four grid points in contact, and the collision force model requires only one distance, thus, the calculations are done at the grid point where the value of the level set function is minimum, such that

$$
\alpha=-\min \phi_{\text {ice }}
$$

Among the five nodes in the contact area on figure 2 , the node $(i, j)$ is the node where the force is evaluated.

For the tangential force calculation, the velocity at node $(i, j)$ is needed. The gravity centre of the body is moving at a velocity $\mathbf{V}_{\mathrm{cg}}$ with an angular velocity $\omega$. If the node $(i, j)$ is located at a distance $\mathbf{r}$ from the gravity centre, then the velocity vector is

$$
\mathbf{V}=\left(V_{x}, V_{y}\right)=\mathbf{V}_{\mathbf{c g}}+\omega \mathbf{r}
$$

This velocity vector is decomposed in normal, $V_{n}$, and tangential, $V_{s}$, components, relative to the wing body. The normal direction is obtained with the gradient of the level set function, $\nabla \phi_{\text {wing }}$ :

$$
n_{x}=\frac{\frac{\partial \phi_{\text {wing }}}{\partial x}}{\sqrt{\left(\frac{\partial \phi_{\text {wing }}}{\partial x}\right)^{2}+\left(\frac{\partial \phi_{\text {wing }}}{\partial y}\right)^{2}}}
$$




$$
n_{y}=\frac{\frac{\partial \phi_{\text {wing }}}{\partial y}}{\sqrt{\left(\frac{\partial \phi_{\text {wing }}}{\partial x}\right)^{2}+\left(\frac{\partial \phi_{\text {wing }}}{\partial y}\right)^{2}}}
$$

In 2D, with $V_{x}$ and $V_{y}$ the Cartesian velocity components, the tangential velocity is obtained by

$$
V_{s}=\left(V_{x} n_{y}-V_{y} n_{x}\right)
$$

The discrete normal force is

$$
F_{n}=k_{n} \alpha
$$

The application point of the force is at the node $(i, j)$. For the tangential force, the tangential displacement during the time interval $\Delta t$ is needed

$$
\Delta \delta=\left(V_{x} n_{y}-V_{y} n_{x}\right) \Delta t
$$

such that

$$
F_{t}=F_{t}^{o l d}+k_{t} \Delta \delta
$$

The magnitude of the tangential force cannot exceed the static friction force, thus

$$
F_{\mathrm{t}}^{\text {new }}=\min \left(\mu\left|F_{n}\right|,\left|F_{\mathrm{t}}^{\text {old }}+k_{t} \Delta \delta\right|\right)
$$

and the force is oriented in the direction of the tangential force.

Once the normal and tangential force components are known, they are projected on the Cartesian axis. The forces are transformed into a linear acceleration, $\mathbf{a}_{c f}$, using the mass of the impinging body, $m$, and into an angular acceleration using the mass moment of inertia of the impinging body, $I_{i c e}$. In 2D,

$$
\begin{aligned}
& a_{x}=\left(F_{n} n_{x}+F_{t} n_{y}\right) / m \\
& a_{y}=\left(F_{n} n_{y}-F_{t} n_{x}\right) / m
\end{aligned}
$$

and

$$
\left(\frac{d \omega}{d t}\right)_{c f}=-\left(F_{x} r_{y}-F_{y} r_{x}\right) / I_{i c e} .
$$




\subsection{Time splitting algorithm}

In the context of the VIC scheme presented in Beaugendre et al. (2011), the collision is taken into account, if needed, by a modification of the moving body velocity. The time splitting algorithm becomes, for each time step:

1. Advection of the vortex by the local velocity field;

2. Computation of the viscous term;

3. Computation of the pressure gradient effects caused by the density variation from fluid to solid;

4. Computation of an intermediate velocity $u^{*}$ from the stream function field computed by solving the linear Poisson equation;

5. Computation of the average linear and angular acceleration inside the solid region from the velocity field;

6. Computation of the solid linear and angular velocities: $\mathbf{V}_{\mathbf{C G}}^{\text {new }}=\mathbf{V}_{\mathbf{C G}}+\mathbf{a} \Delta t$ and $\omega^{\text {new }}=\omega+d \omega / d t \Delta t$

7. Move the solid according to $V_{C G}^{\text {new }}$ and $\omega^{\text {new }}$ to obtain $\left(x_{C G}^{\text {new }}, y_{C G}^{\text {new }}\right)$;

8. If a contact occurs, then subdivide the time step by a factor $N$ and get solid position back to $\left(x_{C G}, y_{C G}\right)$

a. Computation of the acceleration caused by the collision force;

b. Computation of the solid linear and angular velocities: $\mathbf{V}_{\mathbf{C G}}^{\text {new }}=\mathbf{V}_{\mathbf{C G}}+\left(\mathbf{a}+\mathbf{a}_{\mathbf{c f}}\right) \Delta t / N$ and $\omega^{\text {new }}=\omega+\left(d \omega / d t+(d \omega / d t)_{c f}\right) \Delta t / N$;

c. Move the solid according to $V_{C G}^{\text {new }}$ and $\omega^{\text {new }}$ to obtain $\left(x_{C G}^{\text {new }}, y_{C G}^{\text {new }}\right)$;

d. Repeat $N$ times sub step a, b, and c;

9. Computation of the penalisation term.

This algorithm assumes that the velocity field is frozen during the collision. The collision usually occurs for only one or two time steps, and the assumption of a frozen velocity field is close to the calculation reality. The $N$ value should be around ten or twenty to avoid deep overlapping between bodies that could create unphysical strong accelerations. 


\section{RESULTS}

The numerical method is first validated against a well-documented collision test case from the literature. A sphere bouncing on a flat plate in an empty space $(\mathrm{St} \approx \infty)$ is studied with several mathematical models by Thornton, Cummins and Cleary (2011). Then, the numerical method is verified for a particle moving into a viscous fluid and impinging on a cylinder and an aerofoil.

\subsection{Bouncing with negligible viscous effects}

The collision model is validated by comparison with Thornton, Cummins and Cleary (2011) results for a sphere that bounce on a flat plate, without gravity. The sphere is moving freely in a vacuum space; thus, no drag is exerted on it. The sphere radius is $R=25 \mathrm{~mm}$, the density is $\rho=2650 \mathrm{~kg} / \mathrm{m}^{3}$, the Young modulus is $E=70 \mathrm{GPa}$ and the Poisson's coefficient is $v=0.3$. The friction coefficient between the wall and the sphere is $\mu=0.1$. The sphere speed before impact is fixed at $5 \mathrm{~m} / \mathrm{s}$, however, the impact angle $\theta_{i}$ is a variable. The normalized tangential rebound velocity, the normalized angular rebound velocity, the tangential restitution coefficient $e_{t}$, and the normal restitution coefficient $e_{n}$ are defined for a tangential rebound velocity $V_{t r}$ and a normal velocity before impact $V_{n i}$ :

$$
\begin{gathered}
V_{t r}^{\prime}=\frac{2 V_{t r}}{\left(1+e_{n}\right) \mu V_{n i}} \\
\omega_{r}^{\prime}=\frac{2 R \omega_{r}}{\left(1+e_{n}\right) \mu V_{n i}} \\
e_{t}=\frac{V_{t r}}{V_{t i}}, e_{n}=\frac{V_{n r}}{V_{n i}}
\end{gathered}
$$

The following relation defines the dimensionless rebound angle:

$$
\theta^{\prime}=\frac{2 \tan \theta}{\left(1+e_{n}\right) \mu}
$$

Computations are done for impact angle values between $0^{\circ}$ and $45^{\circ}$. The computational domain is rectangular ranging from $(0 \mathrm{~m}, \mathrm{Om})$ to $(2 \mathrm{~m}, 6 \mathrm{~m})$ discretized by $800 \times 2400$ nodes. The sphere motion starts at $(0.05 \mathrm{~m}, 0.03 \mathrm{~m})$. The time steps used for calculation are $0.25 \times 10^{-6}$ $s, 2.5 \times 10^{-6} s$, and $10 \times 10^{-6} s$. The Figures 4,5 , and 6 show the results obtained with the model implemented and the three time steps. The time step influence is small on these 
dimensionless results. However, the time step influence on normal restitution coefficient is significant, ranging from 1.005 for the smallest time step to 1.20 for the largest time step. The dimensionless tangential velocity and the dimensionless angular velocity values on Figures 4 and 5 are close to Thornton, Cummins and Cleary (2011) values, obtained with the same linear spring model but with a different numerical method. For the tangential restitution coefficient, Figure 6, it is important to notice that for small impact angle, the values are very sensitive to the value of the tangential rebound velocity. The tangential velocity is near $0 \mathrm{~m} / \mathrm{s}$ for small impact angles and the results became undetermined for a sphere colliding with $0^{\circ}$ impact angle. This may explain the difference between the results at low dimensionless angles.

For this test case, a collision model that computes only the normal force will fail to predict the rotation of the sphere. It will also not be able to capture the negative tangential rebound velocity after impact and the tangential restitution coefficient will have a constant value of one.

\subsection{Viscous bouncing}

The verification of the numerical implementation of the bouncing model coupled with a flow stream, and gravity is done with an immersed cylinder. A body with a shape similar to a glaze ice shape is placed in front of the cylinder. The case has been presented in details, with ice shedding but without solid collision, in a previous article (Beaugendre et al., 2011). A schematic representation of the dimensionless computational domain $\Omega$ including the cylinder $S^{1}$ and the ice piece $S^{2}$, is sketched on Figure 7. The flow is incompressible inside the rectangular box $[-3,15] \times[-6,6]$. The cylinder diameter is $D=1$; the far field velocity $u=1$ is used as the reference velocity. The ice piece is defined by the area between the curve:

$$
y=0.9-\frac{4}{0.3} x^{2} \text { for } x \in[-0.15 ; 0.15]
$$

and the line of equation $y=0.3$ followed by a rotation of $45^{\circ}$ around the cylinder centre. The fluid density is set to 1 and the solid density is set to 2 . The Reynolds number is 550 . The whole computational domain is meshed with a uniform Cartesian orthogonal grid. After a grid sensitivity study, the grid with spacing $h=1 / 100$ is selected and the non-dimensional time step is set to $0.5 \times 10^{-2}$ for the ice shedding. 
The Figure 8 shows typical ice piece trajectories for two normal spring stiffness ratios. The ratio of tangential spring stiffness over the normal spring stiffness is 0.8235 , and the interface friction coefficient is 0.1 . The normal spring stiffness ratio for a spherical ice shape is related to ice physical properties by equation 8 . However, the ice shape on Figure 8 is not spherical; thus, there are some uncertainties about the normal spring stiffness value that should be used. The results clearly illustrate that the ice piece bounces farther from the cylinder surface for the higher spring stiffness value. The two trajectories are different near the cylinder, but downstream of the cylinder, they almost collapse together.

The second test studied for viscous bouncing is the case of a NACA 0015 with a glaze ice shape in a uniform flow. The computation domain, schematically draw on Figure 9, consists in a rectangular box of $5.5 \mathrm{~m}$ long by $3 \mathrm{~m}$ high. The aerofoil as a $1 \mathrm{~m}$ cord length and the leading edge is located $1.5 \mathrm{~m}$ from entrance. An ice shape is added on the upper side of the aerofoil, near the leading, directly on the aerofoil skin. A uniform velocity of $1.82 \mathrm{~m} / \mathrm{s}$ is imposed at entrance and symmetry boundary conditions are defined at the top and bottom of the rectangular box. The fluid density is $1 \mathrm{~kg} / \mathrm{m}^{3}$ and the ice density is $9.6 \mathrm{~kg} / \mathrm{m}^{3}$. The Reynolds number based on cord length is 2000 and the Froude number is 0.6. The ratio of tangential spring stiffness over the normal spring stiffness is 0.8235 and the interface friction coefficient is 0.1 .

Details of the flow around the aerofoil and ice shape prior to ice shedding are presented on Figure 10. Because of the small size of the computation domain and the small Reynolds number, it does not behave exactly as a free stream flow, but the flow is representative of an aerodynamic flow. The Figure 10 shows the computed vorticity in the computation domain. With the penalisation method, the vorticity is also computed inside the bodies. Most of the vorticity is confined near the bodies, although vorticity is high in the separated flow area behind the ice shape. The streamlines confirm the existence of a separation bubble behind the ice shape. As expected, penalisation ensures that no streamline enters the solid regions.

The figure 11 shows the flow after the ice shedding, when the ice piece is at midway location between aerofoil trailing edge and leading edge. The isocontour of vorticity and the velocity vectors show that the flow is now attached at the leading edge of the aerofoil. Because the ice piece moves more slowly than the flow field, a wake is visible downstream of it. A large separation bubble exists on the upper side of the aerofoil, between the ice piece location and the trailing edge. The wake and separation bubble are a result of the tight coupling between the flow field and the ice piece displacement. 
Three Cartesian grid sizes are used to study the mesh size effect on the solution: $2751 \times 1501$ nodes (coarse), $4126 \times 2251$ nodes (medium), and $6188 \times 3376$ nodes (fine). The time step for the first two grids is $5 \times 10^{-4} \mathrm{~s}$, and for the finest grid, the time step has to be reduced to $2.5 \times 10^{-4} \mathrm{~s}$ for stability reason. The physical time duration of all the calculations is 8s. The Figure 12 shows the computed drag and lift force on the ice piece. The computed aerodynamic coefficients are based on the cord length of the aerofoil. After some oscillations at the beginning of the computation, the drag and lift coefficients become stable. The drag coefficient is a little more sensitive to the grid size than the lift coefficient, the results on the medium size grid being $10 \%$ lower than the ones for the coarse and medium grid. Note that the drag coefficient is only slightly larger than the lift coefficient. The resulting force on ice piece is approximately inclined at $30^{\circ}$ from the horizontal. The ice shape wills roughly slides on the aerofoil surface at the beginning of the shedding motion.

For the three grids, the ice piece trajectories are computed and shown on Figure 13. On the top of the figure the trajectories for the coarse, medium and fine mesh are compared together. On the bottom, the ice piece positions prior to collisions are illustrated. The ice piece first stays close to the surface at the beginning of the motion, until a bounce pushes the ice at a distance from the aerofoil. Because of the gravity, the ice piece falls back on the aerofoil surface several times, meanwhile moving downstream. The computed trajectories are quite sensitive to the grid size, but generally, as the grid gets finer, the ice piece stays closer to the aerofoil surface. Because the ice piece is closer to the aerofoil for the fine mesh, it moves more slowly. Accordingly, the distance travelled by the ice piece is less at the end of the calculation. As can be seen on bottom figure, the ice piece is too close too the surface to rotate. When it starts to rotate clockwise or counter clockwise, one side of the ice piece hits the surface and generates a moment that counters the rotation.

For the iced cylinder and the iced aerofoil, a collision model that does not take into account the tangential force will fail to predict ice piece rotation on impact. With only a normal force computation, the ice bounces away from the surface but the rotations are only induced by the airflow. In reality, a piece of ice that collides with a wall starts to rotate. 


\section{CONCLUSION}

The paper present a collision force model that enables the ice pieces to rebound on the aircraft skin into the airflow. Compare to other CFD works, the model originality consists in the fact that both the normal and tangential forces are computed. The Stoke number, the restitution coefficient, and the contact time are the parameters that govern collision. When an ice piece collides with an aerofoil surface, the ice piece bounces almost instantaneously, with almost no viscous effects, and the speed is conserved at $91 \%$. The propose model is based on linear spring analogy, a model easy to implement in the context of solid motion based on a level set method. The linear spring model assumes that, during contact, the interacting bodies are connected tangentially and normally by linear springs. The model implementation is first verified and validated with a sphere bouncing on a wall at various impact angles, at a constant speed into an empty space. The results agree with literature. Finally, the calculations of ice piece motions close to a cylinder and to an aerofoil are carried for verification of the numerical implementation and stability of the model equation. The bouncing ice pieces behave as expected. Trajectories are extremely sensitive to initial conditions and mesh size. Future works include validation of the model against experimental data, if any available.

\section{Reference}

Al-Khalil, K., Horvath, C., Miller, D. and Wright, W. (1997) 'Validation of NASA thermal ice protection computer codes. III - The validation of ANTICE', in 35th AIAA Aerospace Sciences Meeting and Exhibit, AIAA Paper 97-0051, Reno, Nevada

Appiah-Kubi, P. (2011). U.S. Inflight Icing Accidents and Incidents, 2006 to 2010. Master's Thesis, University of Tennessee.

Ardekani, A. M. and Rangel, R. H. (2007) 'Numerical investigation of particle-particle and particle-wall collisions in a viscous fluid', Journal of fluid mechanics, Vol. 596, pp.437-466.

Beaugendre, H., Morency, F., Gallizio, F. and Laurens, S. (2011)'Computation of Ice Shedding Trajectories Using Cartesian Grids, Penalization, and Level Sets', Modelling and Simulation in Engineering [Online], 2011. Available at: http://www.hindawi.com/journals/mse/2011/274947/ [Accessed 2014].

Cleary, P. W. and Sawley, M. L. (2002) 'DEM modelling of industrial granular flows: 3D case studies and the effect of particle shape on hopper discharge', Applied Mathematical Modelling, Vol. 26, No. 2, pp.89-111. 
Coles, D. M. (2001) 'The microstructure of ice and its influence on mechanical properties', Engineering Fracture Mechanics, Vol. 68, No. 17-18, pp.1797-1822.

Coquerelle, M. and Cottet, G. (2008) 'A vortex level set method for the two-way coupling of an incompressible fluid with colliding rigid bodies', Journal of Computational Physics, Vol. 227, No. 21, pp.9121--9137.

Gammond, P. H., Kiefte, H., Cloutier, M. J. and Denner, W. W. (1983) 'Elastic constants of artificial and natural ice samples by Brillouin spectrosocopy', Journal of Glaciology, Vol. 29, No. 103, pp.403-460.

Glowinski, R., Pan, T. W., Hesla, T. I., Joseph, D. D. and Périaux, J. (2001) 'A Fictitious Domain Approach to the Direct Numerical Simulation of Incompressible Viscous Flow past Moving Rigid Bodies: Application to Particulate Flow', Journal of Computational Physics, Vol. 169, No. 2, pp.363-426.

Green, S. D. (2006) 'A study of U.S. Inflight Icing Accidents', in 44th AIAA Aerospace Science Meeting and Exhibition, AIAA 2006 - 82, Reno, Nevada

Legendre, D., Zenit, R., Daniel, C. and Guiraud, P. (2006) 'A note on the modelling of the bouncing of spherical drops or solid spheres on a wall in viscous fluid', Chemical Engineering Science, Vol. 61, No. 11, pp.3543-3549.

Miller, D., Bond, T., Sheldon, D., Wright, W., Langhals, T., Al-Khalil, K. and Broughton, H. (1997) 'Validation of NASA thermal ice protection computer codes. I - Program overview', in 35th AIAA Aerospace Sciences Meeting and Exhibit, AIAA Paper 97-0049, Reno, Nevada, US

National Transportation Safety Board. (2011) Review of U.S. Civil Aviation Accidents, 20072009, Annual Review NTSB/ARA-11/01 Place, Published

Patankar, N. A. and Sharma, N. (2005) 'A fast projection scheme for the direct numerical simulation of rigid particulate flows', Communications in Numerical Methods in Engineering, Vol. 21, No. 8, pp.419-432.

Petrovic, J. J. (2003) 'Review Mechanical properties of ice and snow', Journal of Materials Science, Vol. 38, No. 1, pp.1-6.

Singh, P., Hesla, T. I. and Joseph, D. D. (2003) 'Distributed Lagrange multiplier method for particulate flow with collisions', International Journal of Multiphase Flow, Vol. 29, pp.495509.

Thornton, C., Cummins, S. J. and Cleary, P. W. (2011) 'An investigation of the comparative behaviour of alternative contact force models during elastic collisions', Powder Technology, Vol. 210, No. 3, pp.189-197.

Wan, D. and Turek, S. (2006) 'Fictitious boundary and moving mesh methods for the numerical simulation of rigid particulate flows', Journal of Computational Physics, Vol. 222, No. 1, pp.28-56.

Wright, W., Al-Khalil, K. and Miller, D. (1997) 'Validation of NASA thermal ice protection computer codes. II - LEWICE/Thermal', in 35th AIAA Aerospace Sciences Meeting and Exhibit, AIAA Paper 97-0050, Reno, Nevada, US 


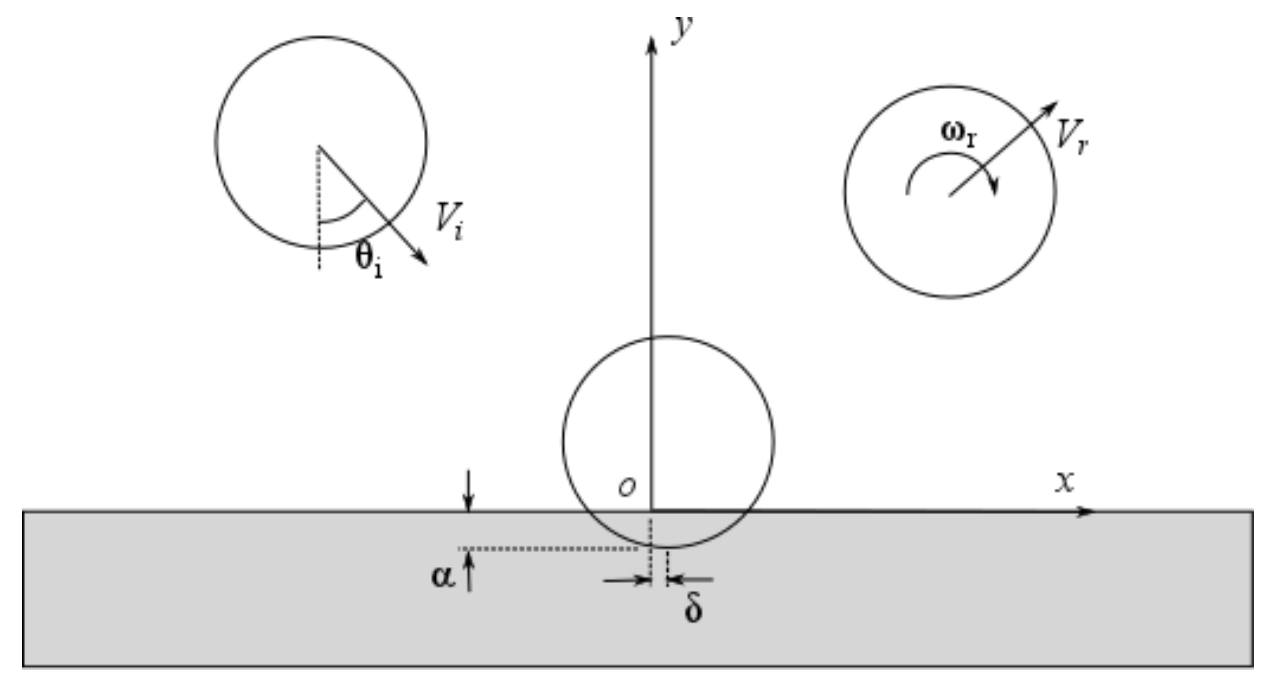

Figure 1 . Sphere bouncing on a wall with a velocity $V_{i}$ and an angle $\theta_{i}$ prior to the contact, without angular velocity. The sphere collides with the wall at point $\mathrm{o}$ and rebounds with a velocity $V_{r}$ and an angular velocity $\omega_{r}$. The sphere overlaps with the wall on a distance $\alpha$ in the normal direction and the relative tangential displacement from the collision point is $\delta$. 


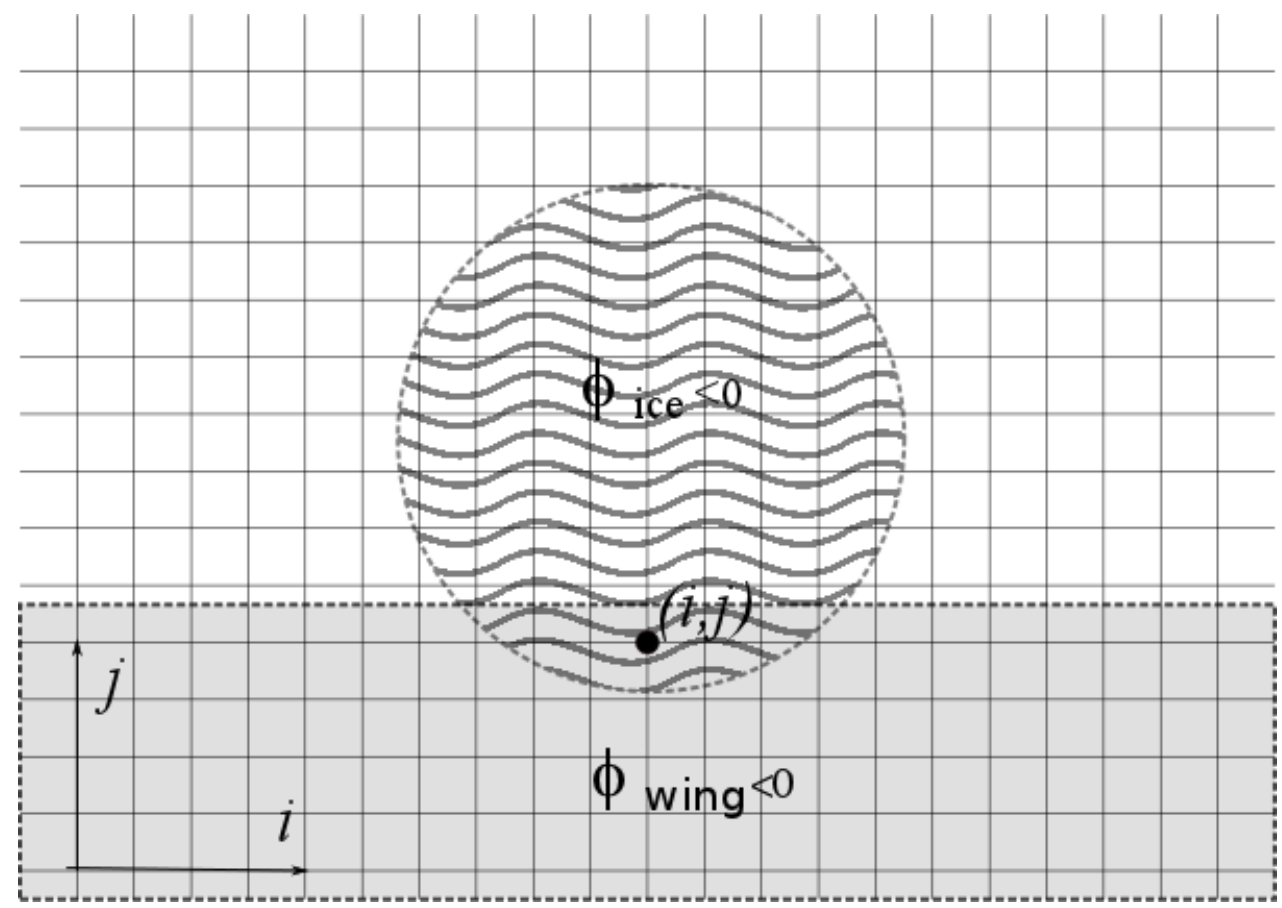

Figure 2: Level set functions and Cartesian grid. Nodes of the Cartesian grid are in the overlap region if $\phi_{\text {wing }}<0$ and $\phi_{i c e}<0$. 


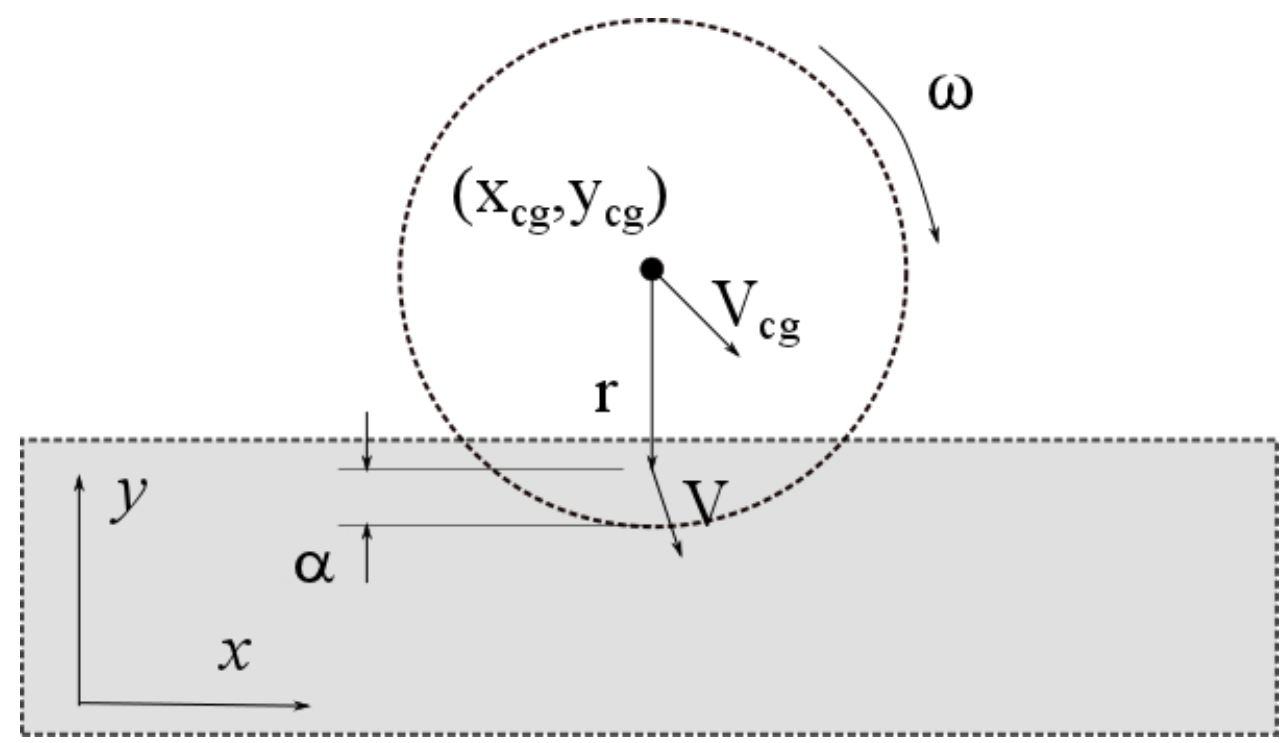

Figure 3 : Variables definition for collision force calculation. The normal distance deformation $\alpha$ is given by the level set function, $\phi_{i c e}$, that is the negative distance between a Cartesian grid point and the body surface. 


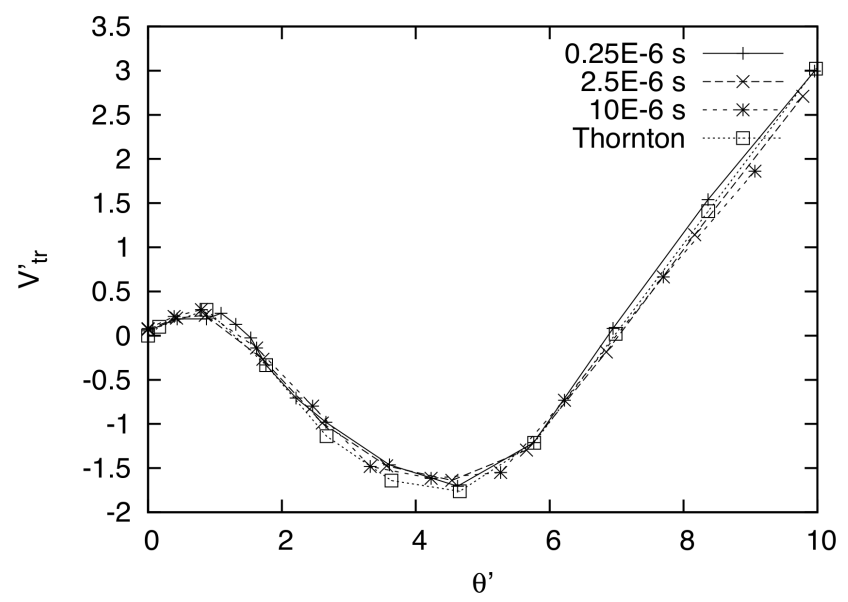

Figure 4. Tangential rebound velocity as a function of the dimensionless rebound angle. Comparison between Thornton results(Thornton, Cummins and Cleary, 2011) and actual computational method for three time steps 


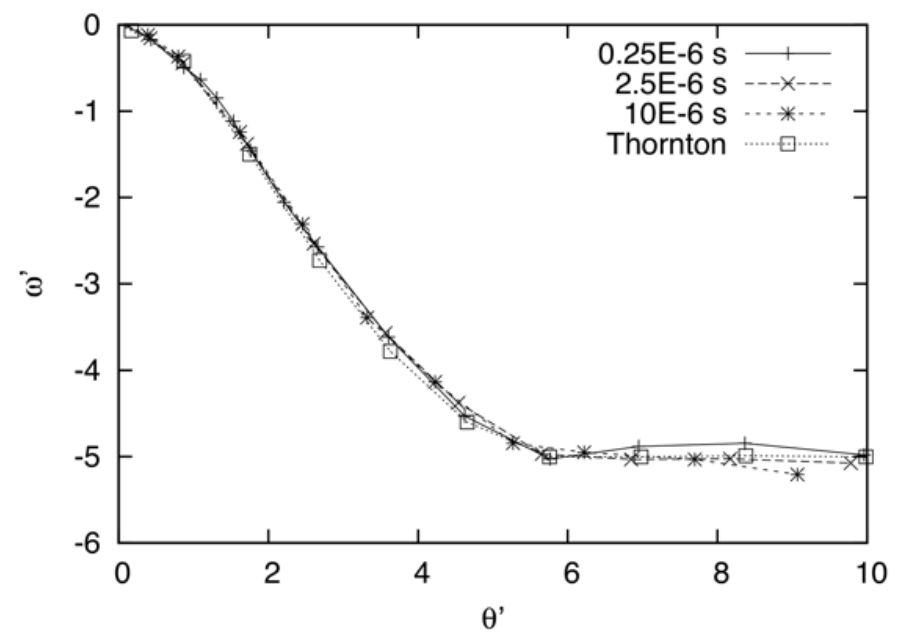

Figure 5. Angular rebound velocity as a function of the dimensionless rebound angle. Comparison between Thornton results(Thornton, Cummins and Cleary, 2011) and actual computational method for three time steps 


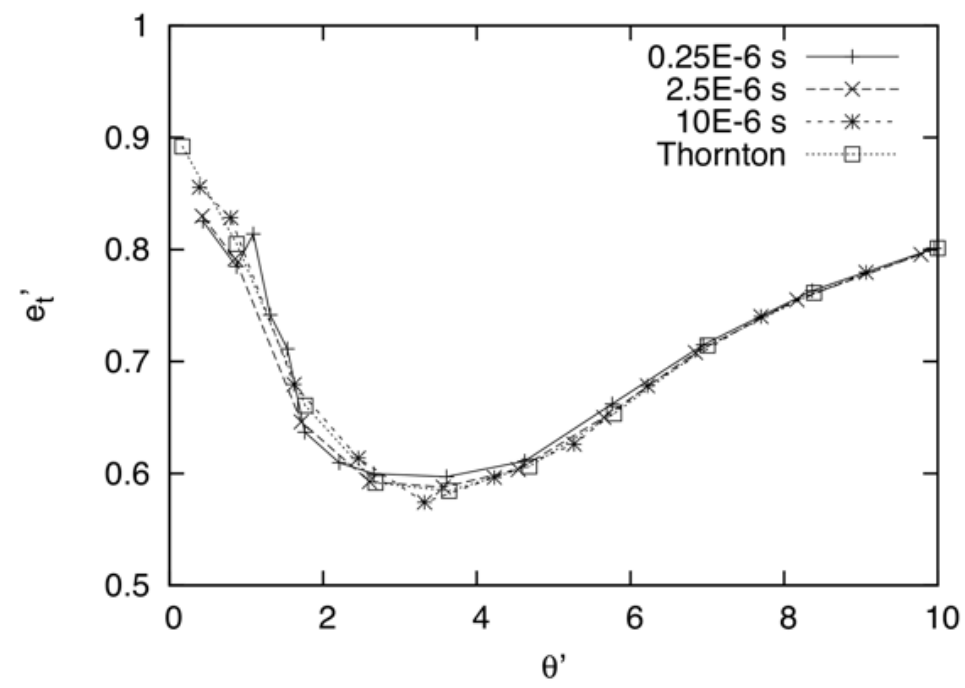

Figure 6. Tangential restitution coefficient as a function of the dimensionless rebound angle. Comparison between Thornton results(Thornton, Cummins and Cleary, 2011) and actual computational for three time steps. 


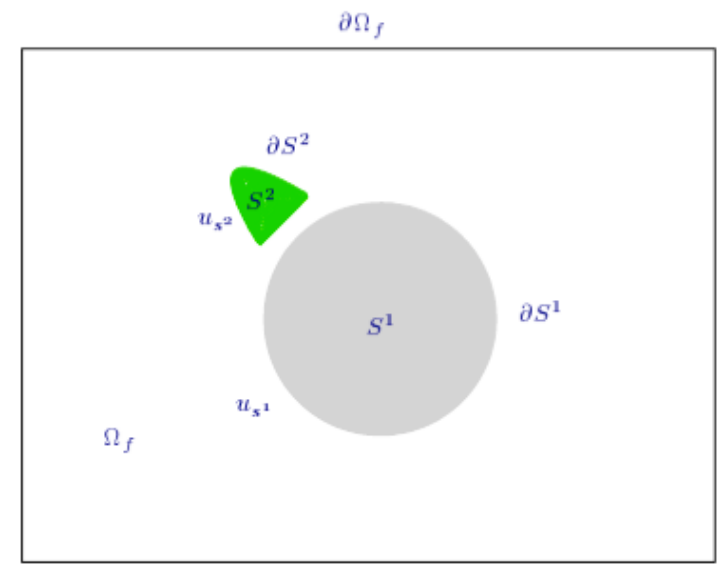

Figure 7. Cylinder and ice shape. The flow is incompressible inside the rectangular box and goes from left to right, with the gravity vector from top to bottom. 


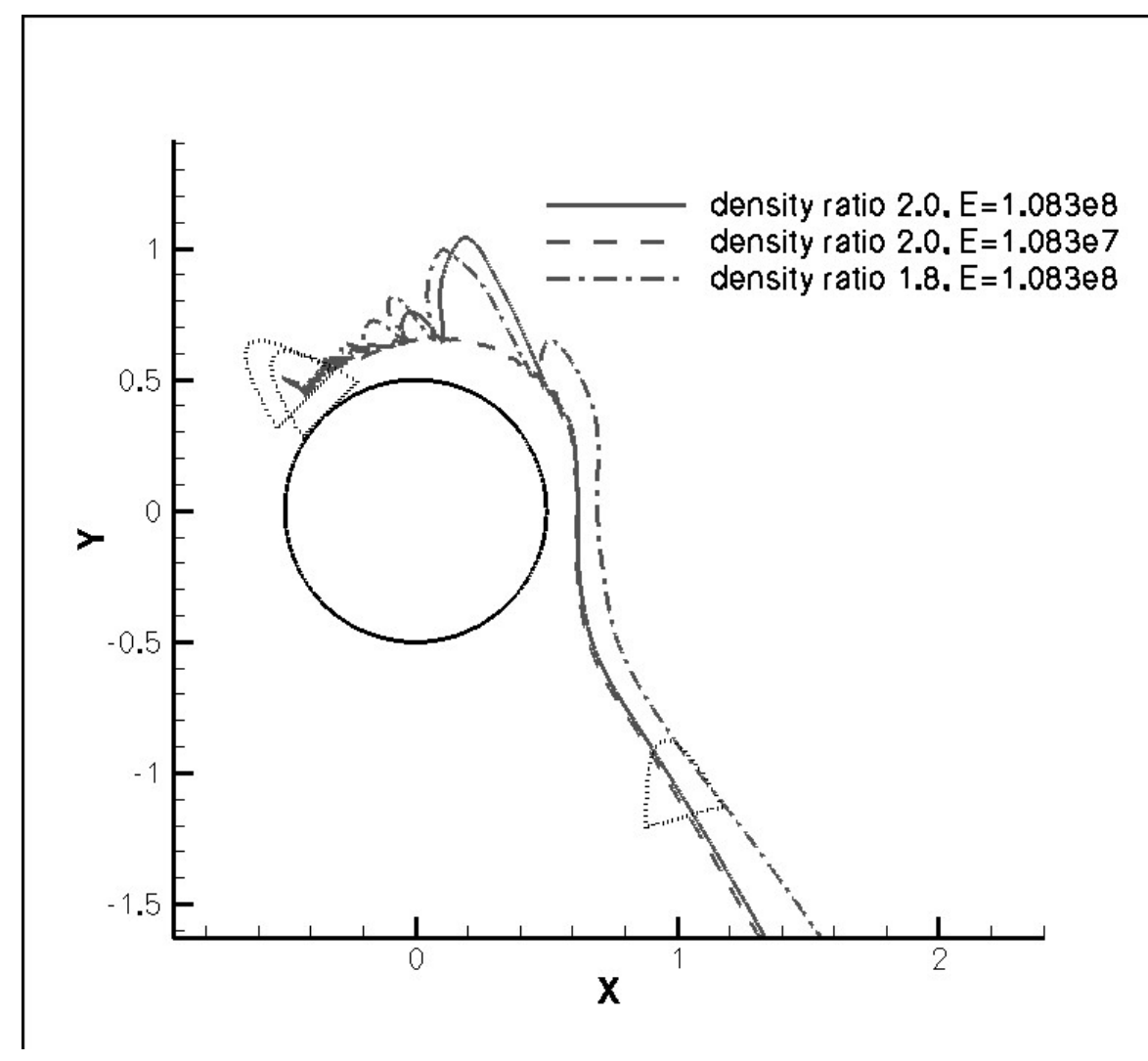

Figure 8. Ice piece trajectories for two normal spring stiffness ratios and two ice density ratios after rebound. 


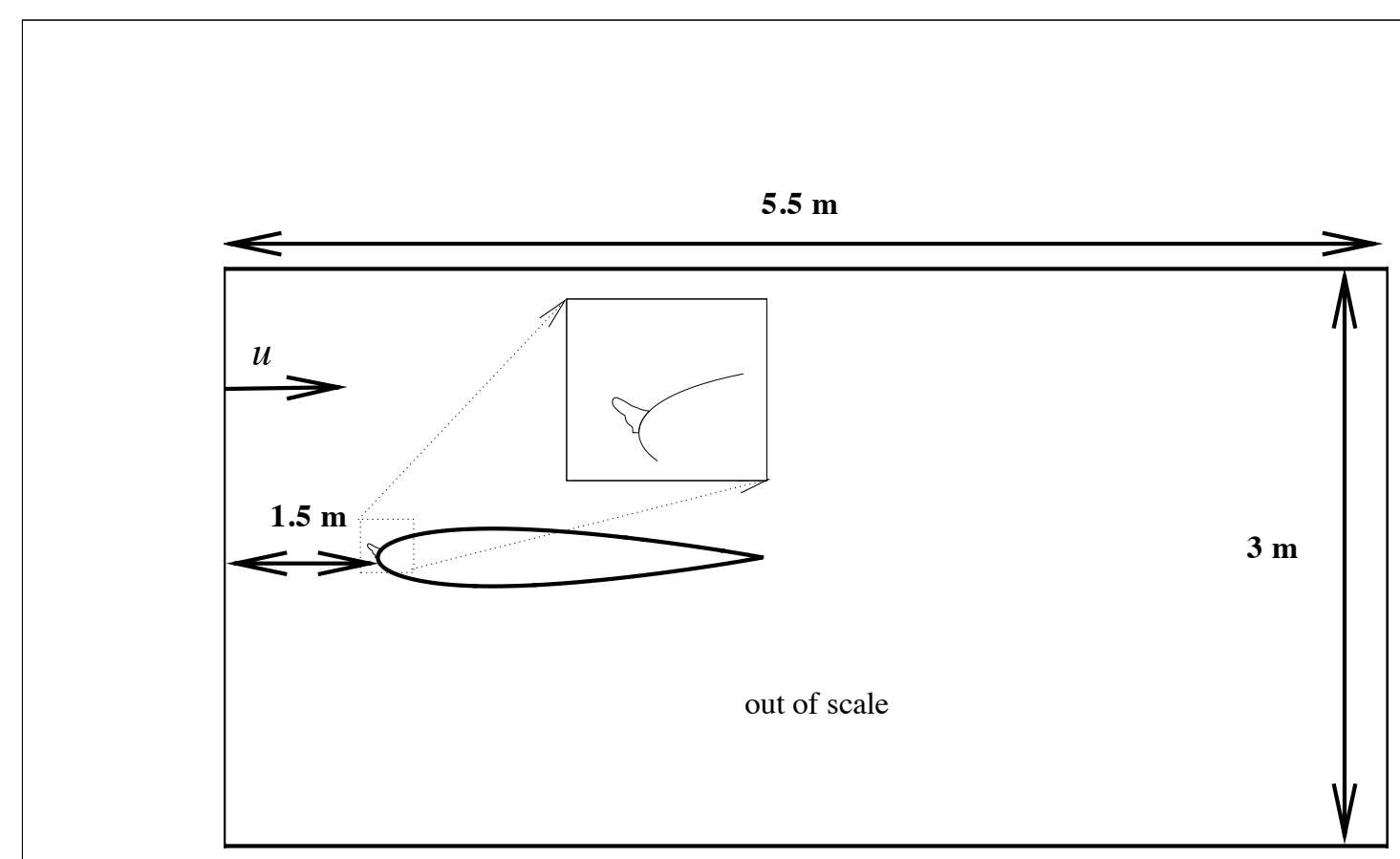

Figure 9 : Computation domain around the $1 \mathrm{~m}$ cord iced aerofoil. The flow is incompressible inside the rectangular box and goes from left to right, with the gravity vector from top to bottom. 


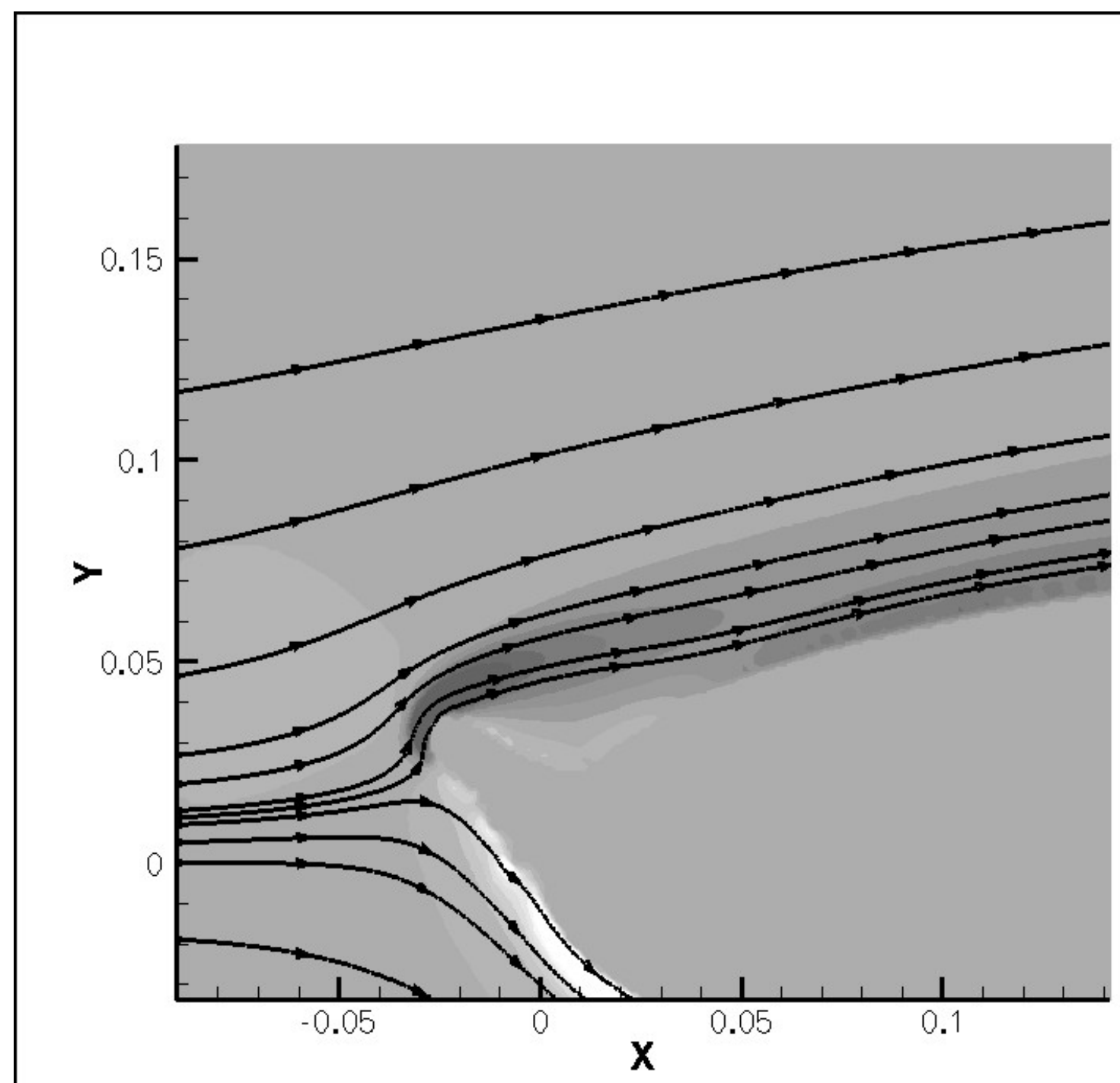

Figure 10 : Flow around ice shape and aerofoil before shedding. The vorticity is computed everywhere with the penalisation method, but most of the vorticity is confined near the body and in the recirculation area. The streamlines confirm the existence of a separation bubble behind the ice shape. 


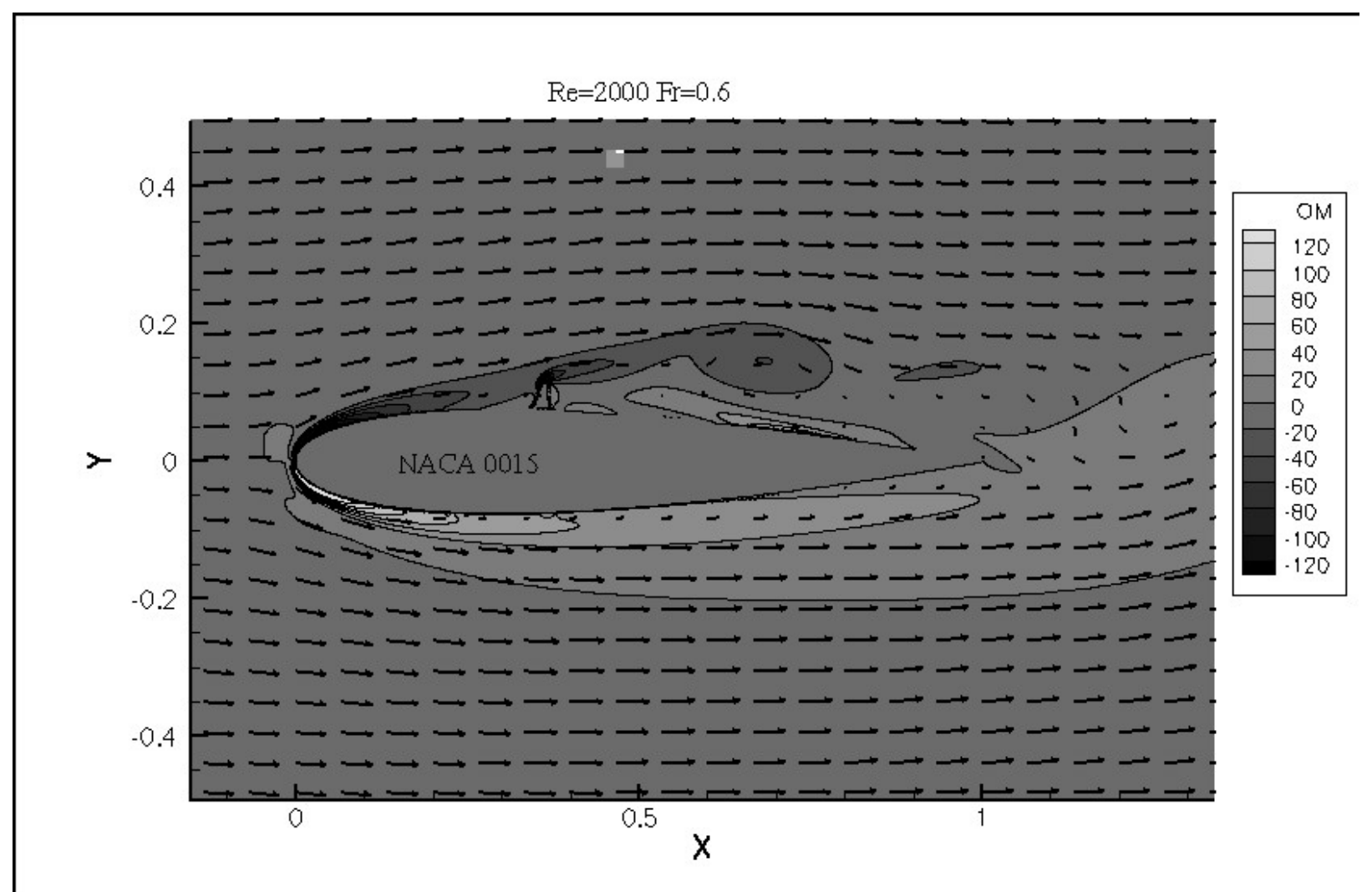

Figure 11 : Flow velocity vectors and vorticity around the aerofoil with ice piece in motion The flow is now attached at the leading edge of the aerofoil but separated after the ice piece, located around $\mathrm{X}=0.4$ on the upper side of the aerofoil. 

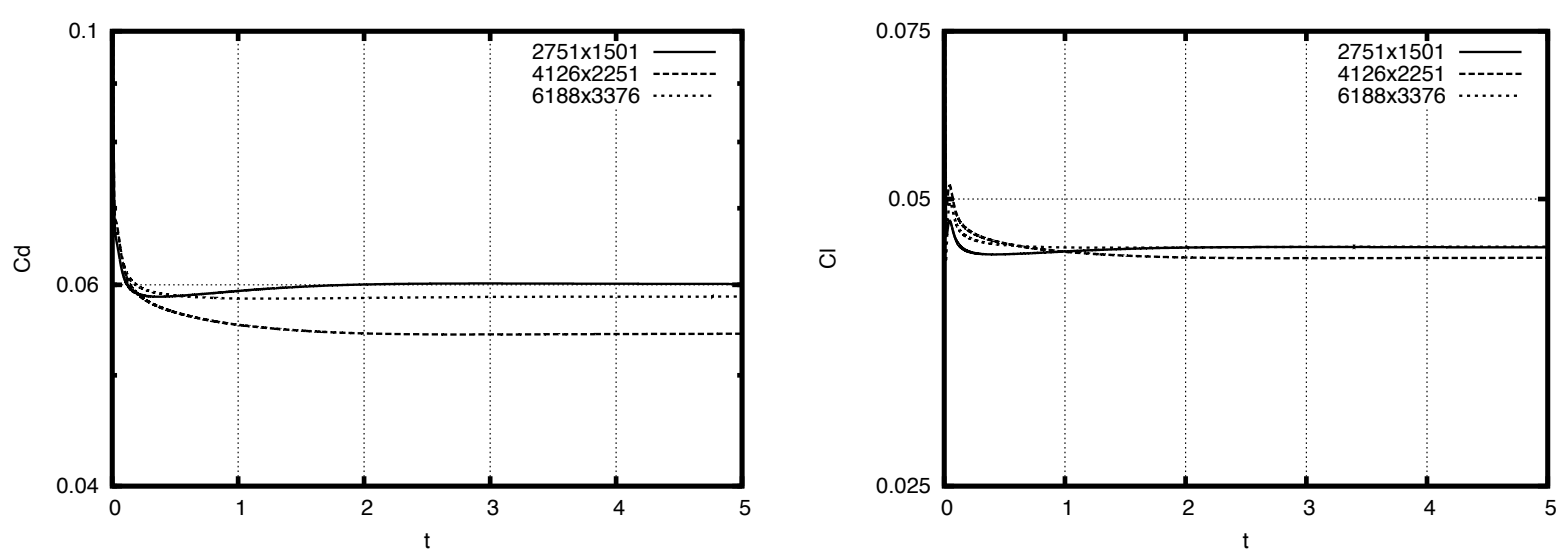

Figure 12 : Effect of grid size on drag and lift exerted on the fixed ice shape for the iced aerofoil. Three Cartesian grid sizes are used: $2751 \times 1501$ nodes (coarse), $4126 \times 2251$ nodes (medium), and $6188 \times 3376$ nodes (fine). The time step for the first two grids is $5 \times 10^{-4} \mathrm{~s}$, and for the finest grid, the time step has to be reduced to $2.5 \times 10^{-4} \mathrm{~s}$ for stability reason. 

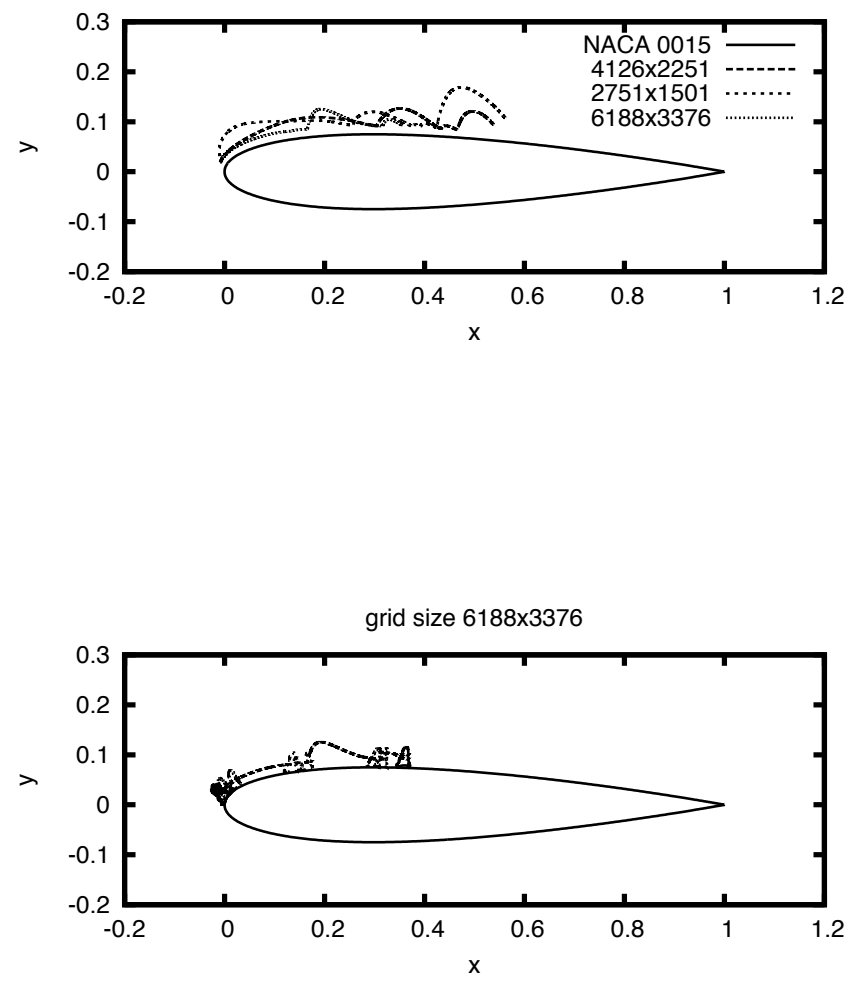

Figure 13 : Ice piece trajectory and bouncing around aerofoil. On the top of the figure the trajectories for the coarse, medium and fine mesh are compared together. On the bottom, the ice piece positions prior to collisions are illustrated for the finest mesh. 